\title{
EGFR NM_005228.3:C.2310_2311insGGC
}

National Cancer Institute

\section{Source}

National Cancer Institute. EGFR NM 005228.3:C.2310 2311insGGC. NCI Thesaurus. Code C98646.

An insertion of three nucleotides, guanine-guanine-cytosine, between position 2310 and 2311 of the coding sequence of the EGFR gene. 